\author{
Revue \\ de /histoire \\ Revue de l'histoire des religions \\ des religions \\ $4 \mid 2015$ \\ Figures de Noé de Gilgamesh au Coran
}

\title{
Un Noé coranisé
}

A Quranized Noah

Viviane Comerro

\section{(2) OpenEdition}

Journals

Édition électronique

URL : http://journals.openedition.org/rhr/8474

DOI : $10.4000 /$ rhr.8474

ISSN : 2105-2573

Éditeur

Armand Colin

Édition imprimée

Date de publication : 1 décembre 2015

Pagination : 623-643

ISBN : 978-2-200-93012-7

ISSN : 0035-1423

Référence électronique

Viviane Comerro, «Un Noé coranisé », Revue de l'histoire des religions [En ligne], 4 | 2015, mis en ligne le 01 décembre 2018, consulté le 19 avril 2019. URL : http://journals.openedition.org/rhr/8474 ; DOI :

$10.4000 /$ rhr.8474 


\section{Un Noé coranisé}

Cet article présente la configuration originale prise par la figure de Noé dans la prédication de Muhammad qui moule les emprunts bibliques dans les stéréotypes coraniques. Le contre-exemple de la sourate 26 qui, échappant à tout comparatisme, voit Noé devenir un prophète sériel dans un corpus coranique clos, nous permettra de réfléchir aux contraintes stylistiques qui pèsent sur la théologie coranique et de là aux conditions de production du texte sacré lui-même.

\section{A Quranized Noah}

This article tackles the original form taken by Noah's figure in the predication of Muhammad, which moulds the Biblical borrowings of Noah into Quranic stereotypes. The counterexample of Surat 26, which defies any comparatist approach and in which Noah's figure becomes a serial prophet in a settled Quranic corpus, will allow us to examine the stylistic constraints of Quranic theology and hence to examine the conditions for the production of the sacred text itself. 
La figure de Noé, telle qu'elle se présente dans le Coran, illustre, parmi d'autres, la nouvelle signification d'un emprunt lorsqu'il est inséré dans un ensemble symbolique différent de ceux qui l'ont précédé. Muhammad s'adresse aux siens par la bouche de Noé en reprenant quelques éléments des récits d'origine biblique dans une configuration originale propre à sa prédication. De la dizaine de péricopes disséminées dans plusieurs sourates du Coran où se trouve récitée l'épreuve de Noé, nous ne ferons que rappeler brièvement, dans la première partie de cet article, les aspects principaux. La seconde partie sera consacrée à l'étude d'une seule sourate, Les Poètes [al-shu'arā'], dans laquelle la figure de Noé, parmi celles d'autres prophètes, fait l'objet d'un traitement sériel. Ce procédé littéraire met en évidence la coranisation d'une figure étrangère coulée dans la prose poétique du Coran dont les parallélismes, les expressions formulaires et les topoi façonnent la vision théologique.

\section{NoÉ dans le Coran}

Dans le Coran, le personnage de Noé est une figure prophétique à l'instar des autres envoyés de Dieu qui font alterner, adressées à leur peuple, sentences de condamnation et annonces de salut. Depuis Noé jusqu'à Muḥammad, la seule figure positive de l'inspiré est, en effet, celle du prophète, envoyé de Dieu (nabī et $r a s \bar{u} l$ ), et celle-ci côtoie d'autres figures négatives comme le devin (kāhin), le sorcier (sāhhir), le possédé des djinns (majnūn), et le poète (shā ir). Autant dire que la manifestation d'une révélation n'était pas quelque chose d'incongru ou de totalement étranger à la culture des Arabes polythéistes du $\mathrm{VII}^{\mathrm{e}}$ siècle, en dehors même de la présence sur leur sol du judaïsme et du christianisme, de mieux en mieux attestée désormais par les historiens, les archéologues et les épigraphistes ${ }^{1}$. En Arabie, les démons ou les djinns aussi étaient une source de révélation, en particulier pour le poète, cet illustre inspiré, auquel ils s'attachaient.

1. Juifs et chrétiens en Arabie aux $v^{e}$ et $V I^{e}$ siècles : regards croisés sur les sources [Actes du colloque organisé à Paris en novembre 2008], éd. Joëlle Beaucamp, Françoise Briquel Chatonnet et Christian Julien Robin, Paris, Centre de recherche d'histoire et civilisation de Byzance (Monographies 32), 2010. 
Dans le Coran, la figure noachique (43 occurrences) est centrale dans une dizaine de péricopes de longueur variable rattachées à la période mekkoise de la prédication de Muhammad par les exégètes, anciens ou modernes, qui se placent dans la perspective d'un Coran écrit reproduisant, plus ou moins fidèlement, sa récitation orale. Comme il s'agit ici de péricopes, les situer dans la période mekkoise n'exclut pas une révision générale des sourates lors d'une étape postérieure à Médine.

C'est probablement à cette étape postérieure de la genèse du texte que la mention de Noé apparaît dans des listes de personnages bibliques qui incluent parfois Muhammad avec lequel il semble en relation particulière comme respectivement premier et dernier prophète ${ }^{2}$.

Cette importance, toute relative, de Noé est confirmée par le fait qu'une sourate entière (71) lui est consacrée dans laquelle il prêche à son peuple comme Muhammad aux Mekkois : louange du dieu créateur et de ses bienfaits, annonce de la Résurrection et dénonciation des idoles de son peuple Wadd, Suwā', Yaghūth, Ya 'ūq et Nasr, dont l'épigraphie et l'onomastique ont retrouvé quelques traces dans la Péninsule arabique ${ }^{3}$. Cette analogie entre Muhammad et les anciens prophètes se trouve d'ailleurs explicitement dans le discours coranique : Et s'ils ne te croient pas, sache qu'avant eux, ont refusé de croire le peuple de Noé, les 'Ād, les Thamūd, le peuple d'Abraham, celui de Loth et les gens de Madian. Moïse lui aussi a été contesté... ${ }^{4}(22,42-44)$.

La figure de Noé est, en effet, associée à l'un des thèmes les plus anciens de la prédication coranique, celui de peuples d'autrefois anéantis par la colère divine après avoir refusé d'entendre la voix du prophète qui leur était envoyé. Ces péricopes dispersées dans l'ensemble du Coran canonique rassemblent des personnages bibliques, comme Noé, Loth, Moïse et Pharaon, ou des peuplades qui hantent le passé de la Péninsule arabique comme le peuple mythique

2. Voir Viviane Comerro, «La nouvelle alliance dans la sourate al-Mā'ida », Arabica 48 (2001), 285-314, p. 295-297.

3. Cf. Christian Robin, Encyclopédie de l'Islam $2^{\mathrm{e} e ́ d . ~(S u p p l e ́ m e n t), ~ « ~ W a d d ~ », ~}$ «Yaghūth», «Ya'ūq ».

4. Wa in kadhdhabū-ka fa-qad kadhdhaba qabla-hum qawmu Nūhin wa 'Ādun wa Thamūdu wa qawmu Ibrāhīma wa qawmu Lūṭin wa așhābu Madyana wa kudhdhiba Mūsā... Q 22, 42×44. 
des 'Ād (déjà mentionné dans la poésie antéislamique), les Thāmud, les Tubba' du Yémen ou encore les « Gens du bois touffu ${ }^{5}$ ».

Notons aussi que le sort de ces anciens peuples annonce, tantôt explicitement tantôt implicitement, l'Heure inéluctable de la destruction de la création qui précédera le Jugement final universel (voir par exemple la sourate 54 al-Qamar). Dans une vision télescopique du passé et de l'avenir, les grandes eaux du châtiment sont associées au feu de l'Enfer : De ce qu'ils ont péché, ils ont été noyés, puis introduits dans le feu $\mathrm{u}^{6}$ tandis que Noé prie pour qu'aucun mécréant ne soit laissé sur la terre ${ }^{7}$.

Ce thème des peuples anéantis par la colère de Dieu a donc un caractère syncrétiste : il mêle aux récits venus de la littérature biblique d'autres récits venus du passé des Arabes et se nourrit, de plus, d'une veine apocalyptique jaillie dans la littérature juive du Second Temple puis transmise dans les milieux chrétiens. Nous apprendrons ensuite comment l'exégèse islamique dans son amplification narrative vient parfois conforter et parfois bouleverser une théologie coranique lapidaire le plus souvent hostile à l'intercession des prophètes ${ }^{8}$.

\section{Les éléments empruntés à la littérature biblique}

Si les récits sur Noé relèvent de scènes-types ou de topoi qui caractérisent à la fois les péricopes sur les peuples anéantis et la propre prédication de Muhammad à son peuple, ils incluent cependant des éléments empruntés à la littérature d'origine biblique par des voies qui nous demeurent toujours inconnues. Communication orale entre individus appartenant aux diverses communautés de l'Arabie plurireligieuse du $\mathrm{VII}^{\mathrm{e}}$ siècle ? Ou comité de scribes réécrivant des textes antérieurs? Le débat fait rage chez les chercheurs.

D'une manière générale, le Noé prédicateur dont les avertissements sont rejetés par un peuple incrédule, si présent dans la représentation coranique, nous vient de la aggada rabbinique et non de la Genèse où ce thème est absent ${ }^{9}$.

5. Așhāb al-Ayka, voir par exemple $Q 26,176$.

6. Mimmā khațī'āti-him ughriqū fa-udkhilū nāran $(71,25)$.

7. $Q 71,26$.

8. Voir dans cette même livraison de la $R H R$ l'article de Youssef Taharraoui.

9. Voir dans cette même livraison de la RHR l'article de Jules Danan, « Noé et le Déluge dans la aggada rabbinique ». 
Le motif du déluge associé à Noé est pourtant retravaillé dans le discours coranique. Le mot lui-même, tŭfă $n^{10}$, n'apparaît qu'une seule fois dans les récits sur Noé $(29,14)$ et une autre fois dans les récits sur Pharaon $(7,133)$ où il désigne l'un des signes-châtiments qui frappent l'Égypte. Il n'a donc pas le caractère d'un fléau dévastant une humanité toute entière corrompue, mais celui d'une inondation locale anéantissant le peuple de Noé sans autre précision, comme est anéanti celui de Loth, ou de Pharaon, de Hūd, de Șāliḥ, ou de Shu 'ayb. Dans d'autres péricopes, il est fait mention de vagues hautes comme des montagnes (11, 42-43), d'une eau torrentielle et de sources qui jaillissent de la terre (54,11-12), mais aussi d'un four ${ }^{11}$ bouillant (11, 40 et 23,27$)$, une image empruntée à la tradition juive ${ }^{12}$.

Si la mention de l'arche est plus fréquente, le terme qui la désigne, fulk ou safina, signifie en arabe tout simplement « bateau » et ne rappelle donc pas le tābūt coranique de Moïse $(Q 20,39)$, contrairement à l'hébreu. Dans la sourate al-Qamar, l'arche est simplement désignée par métonymie pour préserver l'homophonie poétique de la fin des versets sur le son $r$ : dhat alwāh wa dusur, «celle [faite] de planches et de clous» $(54,13)$. En revanche, lorsqu'elle vient à s'échouer après le retrait des eaux jaillies de la terre et du ciel, une localisation précise nous est fournie $(11,44)$ : le Jūdī dans le nord de l'Irak, qui était devenu un lieu de pèlerinage des chrétiens orientaux au monastère de l'arche ${ }^{13}$.

Dans le texte biblique de Genèse le chargement de l'arche est précis : Dieu ordonne à Noé d'embarquer un couple de tout être vivant ou, dans la seconde version, sept couples de tout animal pur

10. Ce terme n'est pas d'origine arabe, il a été rapproché du syriaque țufono, de l'hébreu rabbinique tufonah et du mandéen tufanya, voir Arthur Jeffery, Foreign vocabulary of the Qur'ān, Orient Institut Baroda, 1938, p. 207. Les commentateurs du Coran lui donnent le sens d'inondation, mais aussi d'épidémie.

11. Tannūr : terme que l'on trouve dans de nombreuses langues sémitiques, mais aussi en avestique et en pehlevi, voir Jeffery, Foreign vocabulary, p. 92-95

12. H. Speyer, Die Biblischen Erzählungen im Qoran, Hildesheim, Zürich, New York, Georg Olms, 1988, p. 103 ; voir aussi le déluge dans I Hénoch, 89, 3 : «L'eau se mit à bouillonner et à s'élever de la terre », La Bible. Écrits intertestamentaires, Paris, Gallimard (Pléiade), 1987, p. 579.

13. Voir dans cette même livraison de la RHR Muriel Debié, « Noé dans la tradition syriaque : une mer de symboles : » : selon la Peshitta, l'arche s'est posée sur le mont Qardu (Ğūdi Dagh) et non sur le mont Ararat; sur l'identification du mont Qardu/Gordyene au mont Ararat, voir Michael E. Stone, «Mount Ararat and the Ark », Noah and His Books, éd. Michael E. Stone, Aryeh Amihay, Vered Hillel, Leyde, Brill - Atlanta, Society of Biblical Literature, 2010, 307-316, p. 309. 
et un seul couple de tout animal impur pour en perpétuer la race. Le texte coranique quant à lui est succinct dans son expression formelle : «De tous les couples, un couple $»^{14}(11,40 ; 23,27)$, mais on voit que le commandement est plus large dans son contenu.

Enfin la longévité de Noé, 950 ans nous dit Genèse $(9,29)$, trouve son parallèle exact dans le Coran : "Il demeura dans [son peuple] mille ans moins cinquante années $»^{15}(29,14)$.

Le récit de la sourate 11 (25-48) est particulièrement intéressant puisqu'il comporte un élément dont on n'a trouvé aucun parallèle narratif en dehors du Coran, bien qu'il ait été rapproché d'un passage du livre biblique d'Ézéchiel $(14,14-16)$ : « ...et qu'il y eut ces trois hommes [Noé, Daniel et Job] dans ce pays, par ma vie, oracle du Seigneur Yahvé, ils ne pourraient sauver ni fils, ni filles, eux seuls seraient sauvés, mais le pays deviendrait un désert ».

Dans la péricope de la sourate 11, Noé se voit, en effet, pourvu d'un fils qui refuse de rejoindre les siens. Au moment où Dieu fait voguer les gens de l'arche au milieu de vagues hautes comme des montagnes, Noé interpelle son fils, resté à l'écart : $O$ mon enfant, monte avec nous, ne sois pas parmi les mécréants. Il répond: je vais me réfugier sur une montagne qui me préservera de l'eau. Et Noé : nulle protection contre le commandement de Dieu, sinon sa miséricorde; la vague s'interpose entre Noé et son fils et il fut du nombre des noyés (11, 42-43). Alors Noé interpelle Dieu : Mon fils fait partie des miens! Mais Dieu répond : il n'est pas des tiens !

Le motif du «fils perdu » peut être considéré d'un point de vue intertextuel comme une mise en récit d'Ézéchiel 14, 16 sans que cela indique pour autant une transmission directe. Ce motif rejoint, de fait, la théologie coranique qui voit l'homme au jour du Jugement, seul devant Dieu, sans sa parenté : Craignez votre Seigneur et redoutez le jour où le père n'aura pas à répondre de son enfant et où l'enfant n'aura pas à répondre de son père en quoi que ce soit... $(31,33)$.

Si le fils de Noé nous est présenté en état de perdition, il en est de même pour son épouse qui trouve son modèle dans la femme du Loth biblique comme l'indique explicitement le verset coranique : Dieu donne en exemple aux infidèles la femme de Noé et celle de Loth, elles étaient mariées avec deux de nos bons serviteurs et

14. min kullin zawjayni thnayn.

15. labitha fï-him alfa sanatin illā khamsina 'äman. 
elles les trahirent toutes deux; cela ne leur servit à rien contre la sanction de Dieu et il leur fut dit d'entrer dans le feu avec ceux qui y entrent! $(66,10)$. Encore une fois, il apparaît que toute alliance de chair doit céder devant l'alliance avec Dieu.

Après cette présentation générale de la figure noachique dans le Coran ${ }^{16}$, qui fait apparaitre une composition tributaire à la fois de la littérature biblique et des stéréotypes coraniques, c'est sur ce dernier aspect que je voudrais insister maintenant en limitant mon étude à la sourate dite «Les poètes » (26). Dans l'ordre du comparatisme, il s'agit d'un contre-exemple : Noé y devient un prophète sériel dans un corpus coranique clos. Il me semble pourtant qu'une telle présentation peut nous aider à réfléchir sur deux questions importantes : les contraintes stylistiques qui pèsent sur la théologie coranique et de là sur les conditions de production du texte sacré lui-même.

\section{Le NoÉ Coranisé de la Sourate $A L-S H U^{*} A R \bar{A}$}

\section{La composition de la sourate}

La sourate Les Poètes comporte 227 courts versets, ce qui lui donne une ampleur dont ne témoigne pas exactement son classement à la $26^{\mathrm{e}}$ place dans la version canonique du texte. La sourate constitue-t-elle une unité littéraire ? La tradition musulmane la situe dans la période mekkoise de la prédication de Muhammad tout en émettant parfois l'hypothèse d'une inspiration médinoise des quatre derniers versets. Ce type d'indications peut être interprété comme la reconnaissance d'un travail de compilation et de rédaction effectué sur la récitation orale, soit à des fins d'agencement liturgique soit à des fins de conservation d'une écriture. Que ce travail ait été effectué du vivant du Prophète ou après sa mort est une question débattue aujourd'hui parmi les chercheurs.

$\mathrm{Du}$ point de vue d'Angelika Neuwirth, la sourate 26 a une composition tripartite et se rattache à un ensemble de sourates de la

16. Pour une présentation plus développée, voir Erica Martin, «The Literary Presentation of Noah in the Qur'ān », Noah and His Books, éd. Michael E. Stone, Aryeh Amihay, Vered Hillel, Leyde, Brill - Atlanta, Society of Biblical Literature, 2010, 253-275, p. 253-269 et 273-275. 
période mekkoise tardive ${ }^{17}$. On peut considérer effectivement qu'une introduction (v. 1-9) et une argumentation finale (v. 192-227) l'une et l'autre de nature polémique, viennent enserrer un long récitatif central (v. 10-191) groupant un ensemble de péricopes qui illustrent la confrontation des prophètes anciens avec leurs opposants. Un tel découpage est adéquat, avec cependant quelques réserves sur l'homogénéité de la dernière partie.

La recherche orientaliste a parfois considéré la dernière partie de la sourate (v. 192-227) comme un passage initialement autonome. On a discuté aussi du statut du verset 197, mekkois plus tardif ou médinois ${ }^{18}$, car il concerne la reconnaissance par les docteurs des Fils d'Israël de la révélation de Muḥammad : N'est-ce pas pour eux [les opposants] un signe que les savants des Fils d'Israël connaissent [ce qu'a fait "descendre »le Seigneur des mondes]?

On a aussi émis l'hypothèse que le dernier verset (227) ou les deux derniers versets (227-228) qui viennent moduler la sévère critique des poètes portée par les versets précédents (224-226), pourraient être un ajout médinois ${ }^{19} \mathrm{du}$ fait que certains poètes, dont le célèbre Hiassān b. Thābit, se seraient mis alors au service de la nouvelle religion : Et les poètes, ce sont les égarés qui les suivent, ne vois-tu pas qu'ils divaguent dans toute vallée et qu'ils disent ce qu'ils ne font pas? Excepté ceux qui ont la foi et font de bonnes cuvres et se souviennent de Dieu continûment...

On a aussi mis en lumière le raccord entre deux corpus prophétiques différents, celui plus développé narrativement concernant Abraham et Moïse $(26,10-104)$ et celui plus stéréotypé concernant les autres prophètes $(26,105-191)^{20}$.

Quoi qu'il en soit de l'agencement de ses parties ou de son histoire rédactionnelle, la sourate, dans sa forme achevée, offre une composition formelle extrêmement cohérente par ses parallélismes

17. «Two faces of the Qur'ān: Qur'ān and Muṣhaf», Oral Tradition, 25/1 (2010), 141-156, p. 148.

18. Voir Régis Blachère qui discute la position de Nöldeke, Le Coran, Paris, Maisonneuve et Larose, 1966, p. 402 ; et Théodor Nöldeke, Friedrich Schwally, Gotthelf Bergsträsser, Otto Pretzl, Geschichte des Qorāns, I-III, Hildesheim, G. Olms, 1970, I, p. 126-129.

19. Blachère, Le Coran, p. 403, note 227.

20. Erica Martin, "The Literary Presentation of Noah in the Qur'ān», p. 261-263. 
phoniques, morphosyntaxiques ou formulaires. D'un verset à l'autre, se font écho des rimes plutôt ordinaires en $\bar{\imath} n / \bar{\imath} m / \bar{\imath} l / \bar{u} n / \bar{u} m$, les mêmes mots et des expressions formulaires (qui se retrouvent pour certaines dans d'autres sourates constituant une «intertextualité coranique $»^{21}$ ). De plus, le refrain parénétique qui sépare diverses sections de la sourate lui est propre :

Il y a en cela un signe mais la plupart d'entre eux ne croient pas, ton Seigneur est pourtant puissant et miséricordieux ${ }^{22}$ (v. 8-9 ; 67-68 ; 103-104 ; 121-122 ; 139-140 ; 158-159; 190-191).

Cette forte structuration de la sourate n'empêche pas une variation libre, une improvisation originale, à l'intérieur de l'une ou l'autre de ses parties. Sur le plan sémantique, deux thèmes sont étroitement associés : celui du châtiment, terrestre ou eschatologique, des peuples ignorant l'appel des envoyés de Dieu, et celui de la nature de la révélation, véridique ou mensongère selon sa source.

Précédant le long récitatif central sur les peuples anéantis par la colère de Dieu, les 9 premiers versets forment une introduction dont la limite est bien marquée par la présence du refrain cité cidessus. Ils évoquent l'angoisse du Prophète devant l'opposition que rencontre sa prédication, et la réponse de Dieu par des «signes », signes-versets de l'Écriture, signes de la fécondité de la création, ou signes que rappelleront les événements de l'histoire du salut rapportés par la suite.

Dès le deuxième verset, nous rencontrons une formulation de ce qui est appelé aujourd'hui «l'auto-référentialité » du Coran et que l'on retrouve dans d'autres sourates ${ }^{23}$ : Voici les versets du livre clair/qui explique clairement ${ }^{24}$.

21. La perception de cette intertextualité est fort ancienne dans l'exégèse, mais son expression moderne est bien spécifiée par Angelika Neuwirth, " "Oral Scriptures in Contact". The Qur'ānic Story of the Golden Calf and its Biblical Subtext between Narrative, Cult, and Inter-communal Debate », Self-Referentiality in the Qur'ān, éd. Stefan Wild, Wiesbaden, Harrassowitz, 2006, 71-91, p. 72.

22. Inna fì dhālika la-āyatan wa mā kāna aktharu-hum mu'min̄in wa inna rabba-ka la-huwa l-'azìzu l-rahīm.

23. Cf. $Q 12,1$ et 28, 2 qui reprennent exactement la même formule ; en 15, 1 et 27,1 : les termes kitāb et qur'ān sont associés. Le Kitāb mubīn renvoie à ces écritures divines où est enregistré tout ce qui se passe dans l'univers, un thème de la littérature apocalyptique juive et chrétienne qui trouve un écho explicite en $Q$ 6,59 et 10,61 .

24. Tilka àyātu l-kitābi l-mubīn. 
La prédication de Muḥammad paraît s'y livrer sous la forme très particulière d'un «livre oral $»^{25}$ à même de pouvoir concurrencer l'autorité des Écritures sacrées des juifs et des chrétiens ${ }^{26}$. À côté d'autres indices de cette performance orale primitive du Coran sur lesquels nous reviendrons, la réalisation du verset 4 est intéressante : In nasha' nunazzilu 'alay-him min al-samā' āyatan fa-zallat a'nāqu-hum la-hā khāọi ‘inn'. L'absence d'accord au féminin du terme khä di 'in a préoccupé les commentateurs classiques et certains se sont résignés à y voir une nécessité de la rime en $-\bar{i} n^{28}$.

Les événements de l'histoire du salut qui nous sont ensuite rapportés font allusion à l'opposition rencontrée par sept envoyés de Dieu antérieurs à Muḥammad, figures bibliques, Moïse, Abraham, Noé, Loth, mêlées à des figures du patrimoine arabe, Hūd, Șāliḥ, Shu'ayb. Les péricopes se déroulent dans un ordre décroissant en longueur : 58 versets pour Moïse ; 35 pour Abraham, et moins de 20 versets pour les suivants (18 pour Noé, 17 pour Hūd, 19 pour Sālih, 16 pour Loth, 16 pour Shu'ayb). Une sorte de décroissance s'observe aussi dans la narration : les deux péricopes sur Moïse et Abraham sont les plus développées et aussi les moins stéréotypées ; les cinq autres sont construites sur un même schéma narratif et la présence de Noé dans cet ensemble n'a pas d'importance particulière. Chaque péricope s'achève sur le châtiment des opposants et se clôt sur le refrain parénétique déjà cité : Inna fì dhālika la-āyatan wa mā kāna aktharu-hum mu'minīn wa inna rabba-ka la-huwa l- 'azīzu l-rahìm.

La dernière partie de la sourate se donne comme un argumentaire en faveur de la vraie révélation et une dénonciation polémique de ses contrefaçons. Une brève évocation vient encore rappeler le thème central du récitatif, le châtiment des mécréants, mais il est cette fois à venir, comme une leçon tirée du passé (200-209). À

25. A « spoken book», William A. Graham, « Orality », The Encyclopaedia of the Qur'ān, éd. Jane D. McAuliffe, I-VI, Leiden, Brill, t. III, p. 585.

26. Neuwirth, «Two faces of the Qur'ān», Oral Tradition, 25/1 (2010), 141-156, p. 144-5.

27. «Si nous voulions nous ferions descendre du ciel sur eux un signe et leurs nuques en seraient courbées », l'accord est au masculin pluriel : et leurs nuques en seraient courbés.

28. Par exemple chez l'un des commentateurs les plus orthodoxes, Abū Muḥammad al-Husayn al-Baghawī, Ma'ālim al-Tanzīl, Beyrouth, Dār al-Fikr, 1985, IV, p. 255 : 'alā wifāq ru'ūs al-āyi li-yakūna 'alā nasaq wāhid. Sur le terme ra's al-bayt, voir Lisān al-'Arab: ra's al-bayt qāfiyatu-hu. 
cette actualisation fait écho une brève trouée nous découvrant la situation de Muhammad au milieu de son clan familial et de ses partisans (214-220).

\section{Moüse}

La première péricope du récitatif (v. 10-68) évoque l'appel de Dieu, le refus de Moïse puis son envoi en compagnie d'Aaron, la confrontation avec Pharaon, le concours avec les magiciens, la défaite de ces derniers et leur soumission, la fuite des Fils d'Israël de nuit, la séparation des eaux de la mer, et le signe final : les uns sont sauvés, les autres noyés. La voix narrative du locuteur divin utilise constamment la forme dialoguée que ce soit entre Moïse et Dieu, Moïse et Pharaon, Pharaon et les magiciens ou les magiciens et Moïse.

Bien que ce récit, tout comme le suivant, déroule une succession événementielle qui lui est propre à l'intérieur de la sourate, il utilise un matériau que l'on retrouvera avec quelques variantes dans les autres péricopes.

Le Innā rasūlu rabbi l-'álamīn ${ }^{29}$ (v.16), employé dans d'autres sourates pour Noé et $\mathrm{Hūd}^{30}$ fait ici écho au innī la-kum rasūlun amīn ${ }^{31}$ (v. $105 ; 123 ; 141 ; 160 ; 176$ ) évoquant le motif de l'envoi par Dieu.

Quant à l'expression rabbi l-'âlamìn, fréquente dans le Coran, elle se retrouve cependant 11 fois dans cette même sourate.

Le Inna la-nā la-ajran ${ }^{32}$ (v.41) des magiciens exprimant la demande d'une récompense terrestre s'oppose au refus de toute récompense de cet ordre de la part des prophètes $(\mathrm{v} .109 ; 127 ; 145 ; 164 ; 170)$.

Le salut divin annoncé par le Wa anjaynā Mūsā wa man ma 'a-hu ajma 'ìn thumma aghraqnā l-ākharīn ${ }^{33}$ (v.65-66) s'énonce presque dans les mêmes termes pour Noé (119-120) et pour Loth (v. 170-172).

Enfin, deux locutions ont leur pendant plus avant : Yawmin ma'lūmm ${ }^{34}$ (v. 38 et 155); Jannātin wa' 'uyūnn $n^{35}$ : (v. 57 et $\left.134 ; 147\right)$.

29. « Nous sommes le messager du Seigneur des mondes ».

30. $Q 7,61$ (Noé) ; 7, 67 (Hūd) ; 7, 104 (Moïse).

31. "Je suis pour vous un fidèle messager».

32. «Y aura-t-il pour nous une récompense? »

33. « Nous sauvâmes Moïse et ceux qui étaient avec lui et nous noyâmes les autres ».

34. «Au jour convenu».

35. «Des jardins et des sources» 


\section{Abraham et le châtiment eschatologique des égarés}

La seconde péricope (v. 69-104) est présentée comme une « information » $\left(n a b a^{\prime}\right)^{36}$ qui prend la forme d'un dialogue entre Abraham et son père/son peuple interpellés sur leur adoration des idoles. Elle se poursuit par une glorification du dieu unique et s'achève, non pas sur l'évocation d'un châtiment terrestre des idolâtres, mais sur celle de la rétribution future : les égarés seront précipités $\left(k u b k i b \bar{u}^{37}\right)$ en enfer d'où monteront leurs récriminations.

L'utilisation du terme «égarés » (la forme en arabe est celle d'un participe actif, ghāwūn) doit attirer notre attention. Les ghāwūn, voués à l'enfer sont d'abord opposés aux muttaqūn, les « pieux » destinés au paradis (v. 90-91). Dans les versets suivants (v. 94-95), ils sont associés aux armées diaboliques : fa-kubkibü fi-hā hum wa l-ghāwūn wa junūd Iblīs ajma 'ūn, «ils seront précipités [en enfer], eux, et les égarés et les armées d'Iblīs, tous ensemble». Les gloses exégétiques hésitent sur le référent du pronom - hum : «eux » qui sont-ils et qui sont les ghāwūn ? Les égarés parmi les hommes? Les démons ? Les djinns? Car les égarés sont aussi ceux qui égarent nous dit ailleurs le Coran des entités prétendument associées à Dieu par les hommes : Rabba-nā hā'ulā'i lladhīna aghwaynā aghwaynā-hum ka-mā ghawaynā ${ }^{38}$.

C'est ce même terme de ghāwūn qui sera utilisé dans les derniers versets de la sourate, consacrés aux poètes : wa l-shu 'arā' yattabi'u-hum al-ghāwūn... (v. 224). Quels sont les égarés qui suivent les poètes se demandent les exégètes ${ }^{39}$ ? Des démons ? Des djinns? Des hommes? Ou les uns et les autres à la fois ? A lam tara anna-hum fì kulli wādin yahīmūn (v. 225), « Ne vois-tu pas qu'ils divaguent dans toute vallée ?» Il semble que le terme wād

36. Bien que le terme de qașaș se soit imposé dans la littérature exégétique pour désigner les récits prophétiques du Coran, ceux-ci peuvent être qualifiés indifféremment dans le texte lui-même par les termes de qașaș, de naba' ou de hadìth comme le rappelle Roberto Tottoli, Biblical Prophets in the Qur'ān and Muslim Literature, London and New York, Routledge, 2002, p. 11-12.

37. Hapax legomenon.

38. « Seigneur, ceux que nous avons égarés nous les avons égarés comme nous mêmes sommes égarés » $(Q 28,63)$.

39. Rudi Paret émet un jugement négatif sur l'hypothèse d'Irfan Shahid qui voit dans les ghāwūn des démons, Der Koran, Kommentar und Konkordanz, Stuttgart, Berlin, Köln, W. Kohlhammer, p. 372. Pourtant cette hypothèse trouve un fondement solide dans l'exégèse classique se rattachant à Mujāhid et à Qatāda ; 'Ikrīma y voit lui des djinns rebelles, Țabarī, Tafsīr, en $Q$ 26, 224. 
«vallée » fonctionne ici comme une syllepse qui associe au sens propre du mot son sens figuré. Ne sont-ce pas les démons et les djinns qui inspirent le poète en Arabie et rôdent dans les vallées ?

\section{Noé et les autres prophètes}

Avec Noé débute un ensemble de récits concernant les prophètes Hūd, Șāliḥ, Lūṭ (Loth) et Shu 'ayb, extrêmement stéréotypés tant dans leur progression narrative que par l'emploi d'expressions formulaires. Ainsi chaque péricope commence-t-elle de la façon suivante :

Kadhdhabat qawmu Nühin al-mursalīn Idh qāla la-hum akhūhum Nühưn a lā tattaqūn Innī la-kum rasūlun amīn Fa-ttaqū Llāha wa 'ațī'ūni wa mā as'alu-kum 'alay-hi min ajrin In ajriya illāa 'alā rabbi l-'ālamīn ${ }^{40}$ (105-109)

Kadhdhabat 'Ādun al-mursalīn Idh qāla la-hum akhü-hum Hūdun... ${ }^{41}$ (123-127)

Kadhdhabat Thamūdu al-mursalīn. Idh qāla la-hum akhü-hum Șälihun... ${ }^{42}$ (141-145)

Kadhdhabat qawmu Lüṭin al-mursalīn Idh qāla la-hum akhūhum Lütun... ${ }^{43}$ (160-164)

Et avec une légère variante :

Kadhdhabat aṣhạ̄bu l-aykati al-mursalìn Idh qāla la-hum Shu'aybu... ${ }^{44}(176-180)$

40. «Le peuple de Noé a démenti les envoyés* Lorsque leur frère Noé leur dit : Ne craindrez-vous pas Dieu ? * Je suis pour vous un messager sûr * Craignez Dieu et obéissez-moi * Je ne vous demande nulle rétribution * car ma rétribution n'incombe qu' au Seigneur des mondes ».

41. «Le peuple de 'Ād a démenti les envoyés *Lorsque leur frère Hūd leur dit : Ne craindrez-vous pas Dieu ? * Je suis pour vous un messager sûr * Craignez Dieu et obéissez-moi * Je ne vous demande nulle rétribution * car ma rétribution n'incombe qu'au Seigneur des mondes ».

42. «Le peuple de Thamūd a démenti les envoyés *Lorsque leur frère Șālih leur dit : Ne craindrez-vous pas Dieu ? ${ }^{*}$ Je suis pour vous un messager sûr * Craignez Dieu et obéissez-moi * Je ne vous demande nulle rétribution * car ma rétribution n'incombe qu'au Seigneur des mondes ».

43. "Le peuple de Loth a démenti les envoyés *Lorsque leur frère Loth leur dit : Ne craindrez-vous pas Dieu ? * Je suis pour vous un messager sûr * Craignez Dieu et obéissez-moi * Je ne vous demande nulle rétribution * car ma rétribution n'incombe qu' au Seigneur des mondes ».

44. «Les gens d'al-Ayka ont démenti les envoyés *Lorsque leur frère Shu'ayb leur dit : Ne craindrez-vous pas Dieu? * Je suis pour vous un messager sûr * 
Et toutes les péricopes s'achèvent sur le refrain déjà cité :

Inna fì dhālika la-āyatan wa mā kāna aktharu-hum mu'minīn wa inna rabba-ka la-huwa l-'azīzu l-rahìm.

De la sorte, le commencement de chaque péricope et sa fin sontils entièrement constitués d'expressions formulaires, une indication certaine du caractère oral de la composition du Coran. On peut noter aussi un des effets théologiques générés par ce style formulaire : l'affirmation coranique de l'unité de la prophétie à travers le temps et l'espace, depuis Noé jusqu'à Muḥammad.

C'est entre le commencement et la fin de chaque péricope que s'inscrit la partie originale qui donne à la prédication de chaque prophète sa spécificité : les arguments échangés entre le prophète et ses adversaires, et la nature du châtiment prennent une couleur différente.

Dans ce noyau « atypique » de la péricope, le peuple de Noé lui reproche d'être suivi par les hommes les plus vils de son peuple ${ }^{45}$ : qālū a nu'minu la-ka wa ttaba 'a-ka l-ardhalūn ${ }^{46}$ (v. 111).

Il est aussi menacé de lapidation : qā lū la-in lam tantahi yā Nūh la-takünna min al-marjuminn ${ }^{47}$ (116). La lapidation dans le Coran est une malédiction à l'adresse du diable ${ }^{48}$ et des démons ${ }^{49}$ tentateurs qui viennent écouter les secrets du ciel et égarent les hommes. Il est aussi par antinomie le châtiment dont sont menacés les vrais inspirés par leurs contradicteurs : il en est ainsi pour plusieurs des anciens prophètes Noé, Shu'ayb ${ }^{50}$, Abraham ${ }^{51}$, Moïse ${ }^{52}$, des messagers anonymes ${ }^{53}$ ou les gens de la Caverne ${ }^{54}$.

Craignez Dieu et obéissez-moi * Je ne vous demande nulle rétribution * car ma rétribution n'incombe qu'au Seigneur des mondes ».

45. Le même reproche est adressé ailleurs à Noé à peu près dans les mêmes termes, et en particulier par l'emploi du même qualificatif ardhal qui n'intervient que dans ce contexte : fa-qāla l-mala'u lladhīna kafarū min qawmi-hi mā narā-ka illā basharan mithla-nā wa mā narā-ka ttaba 'a-ka illā lladhīna hum arādhilu-nā : «Le conseil des notables de son peuple qui était mécréant lui dit : nous ne voyons en toi qu'un homme comme nous et nous te voyons suivi par les plus vils d'entre nous » $(11,27)$.

46. «Croirons-nous en toi alors que ce sont les plus vils qui te suivent?»

47. «Ils dirent : si tu ne cesses pas, Noé, tu seras assurément lapidé».

48. $Q 3,36 ; 15,17 ; 16,98 ; 81,25 ; 15,34$.

49. $Q 67,5$.

50. $Q 11,91$.

51. $Q 19,46$.

52. $Q 44,20$.

53. $Q 36,18$.

54. $Q 18,20$. 
Dans ce passage, la réponse de Noé à ses détracteurs comme son invocation à Dieu (v. 118) sont tissées d'expressions trouvant d'autres occurrences dans le Coran : le iftah baynī wa bayna-hum se retrouve ailleurs dans la bouche de $\mathrm{Shu}^{6} \mathrm{ayb}^{55}$ et le najjin $\bar{\imath}$ wa man ma' $\bar{\imath}$ renvoie au najjinī wa ahlī de Loth dans la même sourate (v. 169) ou à d'autres occurrences ${ }^{56}$.

En revanche, le passage comporte une mention originale, celle de l'arche dans laquelle Noé et les siens seront sauvés : fa-anjaynā-hu wa man ma'a-hu fì l-fulki l-mashhüunn (v. 119). Cependant l'originalité est là encore toute relative. L'expression fulk mashhūn est employée ailleurs pour le navire qui embarque Jonas : idh abqa ilā l-fulk al-mashḥūn ${ }^{58}$. Quant au déluge, il n'est pas ici mentionné comme tel puisque l'engloutissement des adversaires de Noé dans les eaux thumma aghraqnā ba'du l-bāqinn (v.120) se présente comme un châtiment semblable à celui qui atteint des gens de Pharaon thumma aghraqnā $l$-ākharīn (v. 68).

Si l'on reprend les éléments qui viennent d'être exposés, on s'aperçoit que la seule particularité attachée à Noé dans cette péricope c'est d'être suivi par des hommes vils lesquels sont, a contrario, des hommes dans le droit chemin selon la bonne révélation divine. Ce chef d'accusation qui singularise Noé ${ }^{59}$ parmi les prophètes frères de leur peuple et même de Muhammad ${ }^{60}$, trouve pourtant un écho particulier à la fin de la sourate : ce sont les poètes, ces faux inspirés, qui sont suivis par des égarés/égarants (v. 224) à la différence de ceux qui invoquent Dieu.

Quoi qu'il en soit du degré d'originalité manifesté dans la texture linguistique, la variation sémantique est bien présente dans le noyau central de chaque péricope :

55. Rabba-nā iftah bayna-nā wa bayna qawmi-nā bi-l-haqq... : « Seigneur, juge entre nous et notre peuple en vérité » $(7,89)$.

56. 28, 21 (Moïse) ; 66, 11(la femme de Pharaon).

57. «Nous le sauvâmes ainsi que ceux qui étaient avec lui dans l'arche chargée ».

58. «Quand il s'enfuit vers le navire chargé » $(37,140)$ : l'image peut rappeler le récit biblique, lorsque les hommes dans la tempête cherchent à alléger le bateau qui menace de se briser.

59. Le qualificatif de ardhal pour désigner ceux qui suivent Noé n'a que deux occurrences dans le Coran : 26, 111 et 11, 27.

60. Comme le remarque H. Speyer, Die Biblischen Erzählungen im Qoran, Hildesheim, Zürich, New York, Georg Olms, 1988, p. 111. 
Pour les 'Ād, bâtir : a tabnūna bi kulli rì'in āyatan ta 'bathūna wa tattakhidhūna mașāni' la 'alla-kum takhludūna ${ }^{61}$ (v. 128-129); et jouir de la fécondité de la création : amadda-kum bi-an 'āmin wa banīna wa jannatin wa 'uyūn $n^{62}$ (v. 133-134) ; la nature du châtiment n'est pas précisée.

Pour les Thamūd, bâtir encore, mais en sculptant la montagne : tanhitūna min al-jibāli buyūtan färihīn ${ }^{63}$ (v. 149); jouir d'une terre fertile : fī jannātin wa 'uyūnin wa zurū'in wa nakhlin tal'u-hā hadìm $^{64}$ (v. 147-148); et le partage de l'eau avec la chamelle rappelant peut-être un vieux conflit entre nomades et sédentaires ${ }^{65}$ (v. 155-157) ; la nature du châtiment n'est pas précisée.

Pour le peuple de Loth, préférer les hommes aux femmes : $a$ ta'tūna l-dhukrāna min al- 'ālamīn wa tadharūna mā khalaqa lakum rabbu-kum min azwāji-kum bal antum qawmun 'ādūn $n^{6667}$ (v. 165-166) ; c'est une pluie qui les anéantit.

Quant aux gens d'Al-Ayka ${ }^{67}$, il semble s'agir de commerçants auxquels le prophète Shu'ayb recommande demande d'être honnêtes dans leurs transactions : awfü l-kayla wa lā takūnū min al-mukhsirīn wa zinū bi-qistạs al-mustaqīm ${ }^{68}$ (v. 181-182); le châtiment du Jour de l'ombre les anéantit.

\section{Vraie ou fausse révélation}

La dernière partie de la sourate (v. 192-227) se déploie après le refrain qui clôt le récitatif : Inna fï dhālika la-āyatan... inna rabba-ka la-huwa l- 'azīzu l-rahīm. La facture narrative y cède la place à une facture argumentative, une alternance stylistique fréquente dans le Coran, pour illustrer une controverse toujours présente. Cependant,

61. «Bâtirez-vous sur chaque mont un signe d'inanité, des monuments qui vous donneront l'illusion de vivre éternellement? »

62. «Il vous donne des troupeaux et des fils, des jardins et des sources ».

63. «Vous sculpterez avec art des habitations dans la montagne».

64. «Au milieu des jardins et des sources, des céréales et des dattiers chargés de fruits ».

65. Voir A.L. de Prémare, "Umm Qirfa et Salmâ et le mythe des peuples anéantis », Journal asiatique, 1/282 (1994), 19-36, p. 32.

66. «Choisissez-vous dans tout l'univers d'aller aux mâles et de laisser les épouses que votre Seigneur a créées pour vous, ô peuple de transgresseurs ».

67. Al-Ayka: le bois touffu; autre lecture possible : Layka qui devient alors un nom propre, Baghawī, Tanzīl, IV, p. 275.

68. «Faites bonne mensure, ne trichez pas ; pesez sur une balance juste ». 
des échos sonores (rimes) des locutions (v. 217 : Dieu est al- 'azīz al-rahìm) et des motifs (v. 201-202 : châtiment eschatologique à venir ; 208-209 : châtiment des peuples d'autrefois) rappellent les parties précédentes. La situation d'énonciation de la prédication est également évoquée : «Avertis [rudement] les membres les plus proches de ton clan », ordonne le locuteur divin à Muhammad « et avec douceur les croyants qui te suivent » (v. 214-215).

Le discours persuasif tire ici profit de la thématique centrale du récitatif. Il affirme que Muhammad est l'héritier de cette révélation divine manifestée à travers les âges, et que ses opposants seront punis brutalement comme cela a toujours été le cas dans le passé : Nous n'avons détruit aucune cité sans qu'un avertissement ne lui ait été adressé par des prophètes car nous ne sommes pas injustes ${ }^{69}$ (v. 208-209). L'argumentaire en faveur de la vraie révélation prêchée par Muḥammad se développe de la façon suivante : elle est une révélation du Seigneur des mondes (192) qui est descendue avec l'Esprit fidèle dans le cœur du Prophète (193-194); elle s'énonce en claire langue arabe (195) et non dans une langue étrangère (198-199) bien qu'elle soit déjà dans les écrits des Anciens (196) et que les doctes parmi les Fils d'Israël la connaissent (197). Ce n'est pas une révélation des démons (210) qui ont beau tendre l'oreille à l'écoute des secrets du ciel, ils n'en ramènent rien et n'inspirent que les imposteurs (221-223). Et les poètes aussi participent de cette fausse inspiration par leurs divagations et leur entourage d'égarés/égarants (224-225). L'enseignement inféré est le suivant: Muhammad quant à lui est un prophète de Dieu, et non un imposteur ni un poète inspiré par les djinns ou les démons ${ }^{70}$.

\section{Conclusion}

On ne peut qu'insister en conclusion sur la force et la cohérence interne du texte coranique pour évaluer la figure de Noé. Celle-ci

69. wa mā ahlaknā min qaryatin illā la-hā mundhirūn dhikrā wa mā kunnā zāalimīn.

70. Sur le statut de la poésie dans la tradition islamique, voir Claude Gilliot, «Poète ou prophète? Les traditions concernant la poésie et les prophètes attribuées au prophète de l'islam et aux premières générations musulmanes », Paroles, signes, mythes. Mélanges offerts à Jamal Eddine Bencheikh, éd. Foréal Sanagustin, Damas, Institut français d'études arabes, 2001, 331-396. 
ne dépend pas seulement des récits empruntés à la littérature juive et chrétienne, mais obéit à une contrainte créatrice propre à la prédication de Muhammad qui façonne aussi sa théologie. La part de réappropriation, c'est-à-dire la part créatrice, se manifeste dans la poéticité propre au Coran, dans ses parallélismes, ses expressions formulaires et ses topoi venant enserrer des éléments de variation et des développements qui brodent sur les schémas-types. C'est dans ces variations que se trouvent les éléments empruntés à la littérature d'origine biblique.

Cette conclusion peut être discutée dans la mesure où elle fait la part belle à l'auctorialité de Muhammad ${ }^{71}$. La composition du Coran est, en effet, une question qui fait débat dans la recherche orientaliste depuis le $\mathrm{XIX}^{\mathrm{e}}$ siècle. Le texte que nous avons entre les mains est-il exactement ou en majeure partie celui de Muhammad ? Ou bien est-il, plus ou moins, le produit d'un comité de rédaction que l'on situe à des dates différentes sous le califat de 'Uthmān, de Mu'āwiya ou de 'Abd al-Malik ? Qui emprunte à qui ? Muḥammad à des récits qui circulent en Arabie ? À un moine de Syrie qui l'instruit? Aux juifs de Médine ? Ou bien des rédacteurs convertis à la nouvelle religion empruntent-ils à des écritures qu'ils connaissent de par leur appartenance antérieure ?

Il semble pourtant difficile de nier la contrainte stylistique imposée par la prédication coranique à tout discours venu d'ailleurs, et les caractéristiques qui la rattachent à la littérature orale ont depuis longtemps été relevées.

En premier lieu, son contexte de production dans une Arabie marquée, depuis le $\mathrm{v}^{\mathrm{e}}$ siècle, par une illustre tradition poétique, tribale et orale, s'exprimant dans une langue extrêmement élaborée ${ }^{72}$. Nous comprenons, par conséquent, ce que nous dit la prédication

71. Pour une discussion critique de la notion de chronologie coranique, voir Gabriel Said Reynolds, «Le problème de la chronologie du Coran », Arabica 58 (2011), 477-502. L'auteur discute ce qu'il appelle un «axiome des études coraniques» qu'il tente de remettre en cause : «L'idée que nous pouvons réorganiser le Coran suivant l'ordre chronologique selon lequel le prophète Muhammad l'aurait proclamé, est pratiquement un axiome des études coraniques. Cette idée repose sur les convictions que le Coran n'a qu'un seul auteur, qu'il n'a aucun rédacteur, et qu'il reflète l'expérience d'une communauté ayant existé autour de Muhammad, à La Mecque et à Médine, entre 610 et 632. »

72. Sur la transmission et la recension de la poésie ancienne, voir Bruno Paoli, De la théorie à l'usage. Essai de reconstitution du système de la métrique ancienne, Damas, Institut Français du Proche Orient, 2008, p. 51-58. 
coranique elle-même du défi qu'elle représentait pour les Arabes contemporains épris d'éloquence. Comme nombre de tournures archaïques du Coran ont posé problème aux exégètes des générations immédiatement postérieures, ils se sont naturellement tournés vers le corpus poétique antéislamique pour en chercher les significations.

En second lieu, la forme du discours coranique elle-même, sa créativité de type cyclique brodant, en fonction des situations, sur des schèmes narratifs ou discursifs d'une sourate à l'autre, son matériau formulaire et ses versets rimés et assonancés. Des études plus récentes cherchent d'ailleurs à pénétrer les secrets d'une composition orale subliminaire. Angelika Neuwirth décrit une technique de composition relevant d'une analyse microstructurale qui fait apparaître des régularités quantitatives entre groupes de versets dans les sourates les plus anciennes ${ }^{73}$. Georges Bohas quant à lui met en évidence les parallélismes métriques entre différents points de la séquence du discours de certaines sourates. Ces parallélismes ne sont pas des réalisations de mètres poétiques arabes strictement codifiés, mais ils n'en manifestent pas moins le haut degré de poéticité du texte ${ }^{74}$.

En troisième lieu, le statut même du texte tel qu'il s'énonce de façon auto-référentielle : le Coran est un acte de parole qui s'adresse à des auditeurs. Tout lecteur corroborera une telle affirmation devant un texte ouvert régulièrement sur du hors-texte, à savoir des situations conflictuelles qui ne portent pas seulement sur des oppositions de croyances ou de doctrines, mais aussi sur des combats bien terrestres et une législation $a d$ hoc qui renvoie à la vie quotidienne d'une communauté en formation.

On peut aussi partir du constat que fait par exemple l'historienne Florence Dupont ${ }^{75}$, celui de l'absence d'autonomie du texte écrit dans

73. « Two faces of the Qur'ān: Qur'ān and Mușhaf», p. 149-150.

74. "Style formulaire et parallélisme dans le Coran », In the Shadow of Arabic : The Centrality of Language to Arabic Culture, éd. Bilal Orfali, Leiden, Brill, 2011, 399-412 ; «La mesure de la sourate Al-Raḥmān », Langues et Littératures du Monde Arabe, 7 (2007), 53-74.

75. «Le couple écriture-lecture n'est ni une donnée universelle ni une donnée transhistorique $[\ldots]$ La différence fondamentale est qu'à notre époque le livre est le support direct d'un texte; nous écrivons un livre qui va donner forme à notre écriture. Ce n'était pas le cas dans l'Antiquité. Le livre accueillait des énoncés composés selon des règles énonciatives propres : un discours politique, un dialogue philosophique, une série de poèmes de banquet. Ce qui impliquait des 
l'Antiquité qui recueille un énoncé composé dans un autre cadre que celui de la raison graphique. La prédication de Muhammad est certes devenue un livre, un muṣhaf, mais après avoir été énoncée selon des règles énonciatives propres au qur'ān. L'importance d'une tradition de récitation orale du Coran - attestée encore de nos jours que vient souligner l'écriture défective des premiers manuscrits non diacrités et non vocalisés, le fait que ces plus anciens manuscrits aujourd'hui datables du milieu du $\mathrm{VII}^{\mathrm{e}}$ siècle comportent déjà non seulement une séparation marquée des sourates, mais un découpage dans ces unités rythmiques liées au souffle que constituent les versets, témoignent de cette absence d'autonomie du texte écrit.

On peut enfin accorder toute son importance à une information qu'il paraît difficile d'inventer tant elle a été interrogée en tous sens dans la tradition et «travaillée » de façon à réduire l'inquiétude qu'elle soulevait ${ }^{76}$ : le qur'ān n'a été rassemblé dans un muṣhaf qu'après la mort du Prophète. On peut traduire le langage de la tradition dans notre idiome moderne en disant que le texte oral du Coran n'a trouvé sa composition finale que sous le califat de 'Uthmān. Pourtant, les termes même de «texte oral » ou de «composition orale » posent problème justement parce que, dans la performance orale, l'énoncé reste soumis à variation. Cependant, dans le cas de la prédication de Muhammad, une modération s'impose si l'on suppose un agencement progressif des versets dans un qur'ān récité lors des prières quotidiennes de la nouvelle communauté.

On pourrait aussi avancer, selon quelques données traditionnelles, l'affirmation d'une transcription de la révélation du vivant même du Prophète. Cette écriture première dont nous ignorons complètement le volume, mais dont nous connaissons en revanche le caractère défectif était-elle contraignante ? On peut en douter si l'on suit la tradition qui a retenu comme une vérité incontournable le logion prophétique des sept $a h r u f^{77}$ selon lequel le Coran pouvait s'énoncer de sept façons différentes. L'authenticité du logion se mesure ici aux multiples débats qui ont surgi pour accorder un même statut à différentes variantes de la récitation et en refuser d'autres, une

identifications diverses de ces énoncés inscrits dans un livre, indépendamment de cette inscription. », L'Antiquité, territoire des écarts, Albin Michel, 2013, p. 256.

76. Voir Viviane Comerro, Les traditions sur la constitution du muṣhaf de 'Uthmān, Beyrouth, Orient- Institut Beirut, 2012.

77. Unzila l-qur'ān 'alā sab 'ati aḥruf. 
pratique qui s'accorde à la fois avec ce que nous savons de l'histoire du texte coranique et de ses lectures, et avec ce que nous rapportent les anthropologues de la littérature orale.

En bref et pour justifier toutes ces considérations qui nous ont éloignée, en apparence, de la figure de Noé, on peut avancer que c'est dans ce cadre large de la compréhension du Coran que la notion d'emprunt, liée au comparatisme dans l'histoire des religions, peut retrouver aujourd'hui une dynamique nouvelle.

viviane.comerro@orange.fr 